\title{
RADIO MEASUREMENTS OF THE STELLAR PROPER MOTIONS IN THE CORE OF THE ORION NEBULA CLUSTER
}

\author{
Sergio A. Dzib ${ }^{1}$, Laurent Loinard ${ }^{1,2}$, Luis F. Rodríguez ${ }^{2,3}$, Laura Gómez $^{4,5,6}$, Jan Forbrich ${ }^{7,8}$, Karl M. Menten ${ }^{1}$, \\ Marina A. Kounkel ${ }^{9}$, Amy J. Mioduszewski ${ }^{10}$, Lee Hartmann $^{9}$, John J. Tobin ${ }^{11,12}{ }^{\text {, and Juana L. Rivera }}{ }^{2}$ \\ Max Planck Institut für Radioastronomie, Auf dem Hügel 69, D-53121 Bonn, Germany; sdzib@mpifr-bonn.mpg.de \\ ${ }^{2}$ Instituto de Radioastronomía y Astrofísica, Universidad Nacional Autónoma de México Apartado Postal 3-72, 58090, Morelia, Michoacán, Mexico \\ ${ }^{3}$ El Colegio Nacional, Donceles 104, 06020, México, DF, México \\ ${ }_{5}^{4}$ Joint ALMA Observatory, Alonso de Córdova 3107, Vitacura, Santiago, Chile \\ ${ }^{5}$ CSIRO Astronomy and Space Science, PO Box 76, NSW 1710 Epping, Australia \\ ${ }^{6}$ Departamento de Astronomía, Universidad de Chile, Camino El Observatorio 1515, Las Condes, Santiago, Chile \\ ${ }_{8}^{7}$ University of Vienna, Department of Astrophysics, Trkenschanzstr. 17, 1180 Vienna, Austria \\ ${ }^{8}$ Harvard-Smithsonian Center for Astrophysics, 60 Garden Street, Cambridge, MA 02138, USA \\ ${ }^{9}$ Department of Astronomy, University of Michigan, 500 Church Street, Ann Arbor, MI 48105, USA \\ ${ }^{10}$ National Radio Astronomy Observatory, Domenici Science Operations Center, 1003 Lopezville Road, Socorro, NM 87801, USA \\ ${ }^{11}$ Homer L. Dodge Department of Physics and Astronomy, University of Oklahoma, 440 W. Brooks Street, Norman, OK 73019, USA \\ ${ }^{12}$ Leiden Observatory, Leiden University, P.O. Box 9513, 2300 RA Leiden, The Netherlands \\ Received 2016 September 4; revised 2016 November 4; accepted 2016 November 11; published 2017 January 11
}

\begin{abstract}
Using multi-epoch Very Large Array observations, covering a time baseline of 29.1 years, we have measured the proper motions of 88 young stars with compact radio emission in the core of the Orion Nebula Cluster (ONC) and the neighboring $\mathrm{BN} / \mathrm{KL}$ region. Our work increases the number of young stars with measured proper motion at radio frequencies by a factor of 2.5 and enables us to perform a better statistical analysis of the kinematics of the region than was previously possible. Most stars (79 out of 88 ) have proper motions consistent with a Gaussian distribution centered on $\overline{\mu_{\alpha} \cos \delta}=1.07 \pm 0.09 \mathrm{mas} \mathrm{yr}^{-1}$, and $\overline{\mu_{\delta}}=-0.84 \pm 0.16 \mathrm{mas} \mathrm{yr}^{-1}$, with velocity dispersions of $\sigma_{\alpha}=1.08 \pm 0.07 \mathrm{mas} \mathrm{yr}^{-1}, \sigma_{\delta}=1.27 \pm 0.15 \mathrm{mas} \mathrm{yr}^{-1}$. We looked for organized movements of these stars but found no clear indication of radial expansion/contraction or rotation. The remaining nine stars in our sample show peculiar proper motions that differ from the mean proper motions of the ONC by more than $3 \sigma$. One of these stars, V 1326 Ori, could have been expelled from the Orion Trapezium 7000 years ago. Two could be related to the multi-stellar disintegration in the $\mathrm{BN} / \mathrm{KL}$ region, in addition to the previously known sources $\mathrm{BN}$, I and $\mathrm{n}$. The others either have high uncertainties (so their anomalous proper motions are not firmly established) or could be foreground objects.
\end{abstract}

Key words: astrometry - radiation mechanisms: non-thermal - radiation mechanisms: thermal - radio continuum: stars - techniques: interferometric

\section{INTRODUCTION}

The angular resolution of radio interferometers improves proportionally with the longest baseline in the array. With baselines of tens of kilometers, as with the Karl G. Jansky Very Large Array (VLA), angular resolutions of order 0 " 1 are possible around $\nu=10 \mathrm{GHz}$. This enables source positions to be measured to about 0 " 01 even for moderate signal-to-noise detections. In addition, interferometric radio observations are usually phase-referenced with respect to background quasars whose positions are accurately measured in the International Celestial Reference Frame. Thus, although they are not strictly absolute, the positions delivered by radio interferometers at multiple epochs can be directly compared, and accurate proper motions can be measured. Indeed, VLA observations with time separations of several years have been used to measure proper

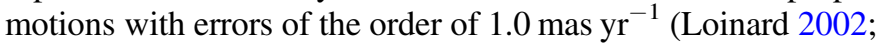
Rodríguez et al. 2003; Dzib et al. 2014).

A significant fraction of Young Stellar Objects (YSOs) are radio emitters thanks to two main mechanisms: thermal bremsstrahlung (free-free) and non-thermal gyrosynchrotron emission. Both classes can be observed with the VLA. Thus, multi-epoch VLA observations can be used to accurately measure the proper motions of YSOs. This is particularly interesting because, unlike optical or near-infrared observations, radio measurements are essentially immune to obscuration by dust.

The Orion Nebula Cluster (ONC) is the nearest region $(d=414 \pm 7 \mathrm{pc} \text {, Menten et al. 2007 })^{13}$ having recently formed massive stars. In the core of the ONC (Hillenbrand \& Hartmann 1998), there are two sub-regions of particular interest: the Orion Trapezium and the Orion $\mathrm{BN} / \mathrm{KL}$ region, which is located behind the ONC. Together they cover an area of a few square arcminutes. This area is also one of the most crowded with YSOs emitting at radio frequencies (i.e., Churchwell et al. 1987; Garay et al. 1987; Zapata et al. 2004; Kounkel et al. 2014; Forbrich et al. 2016). Taking advantage of these characteristics and using multi-epoch observations, the proper motion of several objects were measured by Gómez et al. (2005, 2008). Here we will expand and improve this study by adding recent observations made with the newly expanded VLA at similar frequencies and resolutions as those used by Gómez et al. (2005, 2008). We will use the new proper motions to study the overall Galactic motion of the cluster, its internal kinematics, and to identify radio sources with peculiar velocities. Finally, our study can be

\footnotetext{
${ }^{13}$ Recent results of the Gould's Belt Distances survey (GOBELINS) suggest a smaller distance of $388 \pm 5 \mathrm{pc}$ (Kounkel et al. 2016a). This new value does not affect our results significantly.
} 
Table 1

Trapezium-BN/KL Observations and Final Parameter of Maps

\begin{tabular}{lccc}
\hline \hline Epoch & $\begin{array}{c}\lambda \\
(\mathrm{cm})\end{array}$ & $\begin{array}{c}\text { Synthesized Beam } \\
\theta_{\text {maj }}\left[^{\prime \prime}\right] \times \theta_{\min }\left[^{\prime \prime}\right] ; \text { P.A. }\left[^{\circ}\right]\end{array}$ & $\begin{array}{r}\text { rms Noise } \\
\left(\mu \mathrm{Jy} \mathrm{bm}^{-1}\right)\end{array}$ \\
\hline 1985.05 & 6.0 & $0.43 \times 0.35 ;-15$ & 136 \\
1991.67 & 3.6 & $0.26 \times 0.25 ;-55$ & 77 \\
1995.56 & 3.6 & $0.26 \times 0.22 ;+34$ & 42 \\
2000.87 & 3.6 & $0.24 \times 0.22 ;+3$ & 40 \\
2006.36 & 3.6 & $0.26 \times 0.22 ;-2$ & 58 \\
2011.50 & 5.0 & $0.30 \times 0.27 ;+46$ & 115 \\
2011.56 & 5.0 & $0.47 \times 0.24 ;-47$ & 110 \\
2011.66 & 5.0 & $0.33 \times 0.25 ;-30$ & 102 \\
2012.76 & 4.0 & $0.22 \times 0.20 ;-7$ & 100 \\
2014.17 & 5.5 & $0.44 \times 0.29 ;-38$ & 45 \\
2014.17 & 3.3 & $0.25 \times 0.18 ;-38$ & \\
\hline
\end{tabular}

compared with kinematic studies at optical wavelengths of radial velocities (Kounkel et al. 2016b) and proper motions (Poveda et al. 2005).

\section{OBSERVATIONS}

We searched the VLA archive for deep $\left(<150 \mu \mathrm{Jy}^{\text {beam }}{ }^{-1}\right)$ observations recorded in the most extended A configuration at $\mathrm{C}$ band $(4-8 \mathrm{GHz})$ and $\mathrm{X}$ band $(8-12 \mathrm{GHz})$. The frequencies and configuration were chosen as a compromise between angular resolution (better than 0 . 5 ) and the area covered by the field of view. These two bands are also the most sensitive of the VLA.

Observations with these characteristics were found for the following epochs (see also Table 1): 1985 January 19, 1995 July 2, 2000 November 13, 2006 May 12 (the observations up to this date were previously analyzed by Gómez et al. 2005, 2008), 2011 July 2, 2011 July 24, 2011 August 29 (results of these observation were reported by Kounkel et al. 2014), 2012 October 3 (Forbrich et al. 2016), and 2014 March 3 (archival data reported here for the first time). All these observations used the quasar J0541-0541 as phase calibrator, a source that is at an angular separation of 1.6 from the ONC core. We include an additional epoch, 1991 September 6, to more uniformly cover the time baseline of $\sim 30$ years between the first and last observed date. However, the quasar J0501-019 was used as phase calibrator in this epoch. The angular separation of this quasar from the ONC core is $9^{\circ} .1$. Such a large separation will affect the measured positions for this epoch (e.g., Pradel et al. 2006). In fact, Gómez et al. (2008) estimated a systematic offset in decl. of $\Delta \delta=-0$.'035 for this observation, which we will use here as well.

Observations that were taken prior to the VLA expansion in 2010 were calibrated, edited and imaged in AIPS as described in Gómez et al. (2005, 2008). The remaining epochs were processed similarly using the CASA software (McMullin et al. 2007). Positions of the sources were obtained using a twodimensional Gaussian fit (task "imfit" in CASA). For epochs 2011.36, 2011.50 and 2011.56, in order to obtain a better noise level than that reported by Kounkel et al. (2014), we combined the two recorded sub-bands (each $1 \mathrm{GHz}$ wide and centered, respectively, at 4.5 and $7.5 \mathrm{GHz}$ ). Also, when a given source was detected in several of the three epochs reported by Kounkel et al. (2014), we use a weighted average of the positions, and a time-stamp corresponding to the weighted mean of the corresponding epochs.

\section{RESULTS}

After measuring the positions of the sources in individual epochs, we used them to compute their proper motions. In order to obtain accurate results we restricted our proper motion measurements to those radio sources with at least three detections and a minimum separation between the first and the last detected epochs of 5 years. A total of 92 sources fulfill these requirements. This is more than twice than those previously analyzed by Gómez et al. (2005). From these sources, four are related to the explosive event in the $\mathrm{BN} / \mathrm{KL}$ region and will be discussed in a separate paper by Rodríguez et al. (2017). Proper motions for the remaining 88 sources were obtained using a least-squares fitting to the positions of the sources. Systematic errors of the order of 10 mas were added to the errors given by imfit, in order to obtain a reduced $\chi^{2}$ of one. The systematic errors are expected to be dominated by uncertainties introduced by the interpolation of the phase calibration from the quasar to the targets field. The measured proper motions are listed in columns 3 and 4 of Table 2 and their distribution in the plane of the sky are shown in Figure 1. From our sample, for 14 stars $(16 \%)$ the proper motion measurements are significant above $2-\sigma$ in both directions (R.A. and decl.), for $43(49 \%)$ the proper motion in at least one direction is above $2-\sigma$, while for $31(35 \%)$ the measurements are below 2- $\sigma$ in both directions. To highlight the first two cases in Figure 1 we used different levels of transparency when we draw the arrows. To calculate the transverse velocities corresponding to these proper motions, we use $1 \mathrm{mas} \mathrm{yr}^{-1} \equiv 1.96 \mathrm{~km} \mathrm{~s}^{-1}$ as appropriate for the distance (414 pc; Menten et al. 2007) to the ONC.

We now compare our results with previous results. In the top panels of Figure 2, we show the comparison between our measured absolute proper motion and those obtained by Gómez et al. (2005) for the 35 radio sources that we have in common. While most of the proper motions in R.A. agree within $1 \sigma$, in decl. there is a systematic shift. This systematic shift can be attributed to the different quasar used in the 1991.68 epoch. As we mentioned before, Gómez et al. (2008) found a systematic shift in decl. of $\Delta \delta=-0$.' 035 that was not used by Gómez et al. (2005). To test our hypothesis we measured the proper motion of the same 35 sources without epochs 1985.05 and 1991.68 and compare them with the proper motions from Table 2, this comparison is shown in the bottom panels of Figure 2. In this case we see that the vast majority of the proper motions agree in both R.A. and decl. Thus, we confirm that it is correct to use the $\Delta \delta$ value found by Gómez et al. (2008) for the epoch 1991.68.

From the same top panels of Figure 2, an improvement in the error can be noticed between the old and new measurement of proper motions. These improvements are on average of the order of two. As can be seen in the Appendix, the proper motion errors are expected to decrease with time as $t^{-3 / 2}$, in ideal cases. Thus, by duplicating the time baseline we expect an improvement in errors by a factor of 2.8. Differences between the real improvements and those expected can be due to the non-uniform sampling when observing and to different position errors between the observed epochs.

Menten et al. (2007) used the Very Long Baseline Array (VLBA) telescope to observe four non-thermal YSOs (GMR A, 
Table 2

Proper Motions of the Radio Sources in Orion

\begin{tabular}{|c|c|c|c|c|c|}
\hline \multirow[b]{2}{*}{ VLA Name } & \multirow[b]{2}{*}{$\begin{array}{l}\text { Other } \\
\text { Name }^{\mathrm{a}}\end{array}$} & \multicolumn{2}{|c|}{ Absolute } & \multicolumn{2}{|c|}{ Orion's Rest Frame } \\
\hline & & $\begin{array}{c}\mu_{\alpha} \cos \delta \\
\left(\mathrm{mas} \mathrm{yr}^{-1}\right)\end{array}$ & $\begin{array}{c}\mu_{\delta} \\
\left(\operatorname{mas~yr}^{-1}\right)\end{array}$ & $\begin{array}{c}\mu_{\alpha} \cos \delta \\
\left(\mathrm{mas} \mathrm{yr}^{-1}\right)\end{array}$ & $\begin{array}{c}\mu_{\delta} \\
\left(\operatorname{mas~yr}^{-1}\right)\end{array}$ \\
\hline J053509.76-052128.2 & COUP 342 & $-3.77 \pm 2.96$ & $-1.39 \pm 3.85$ & $-4.84 \pm 2.96$ & $-0.55 \pm 3.85$ \\
\hline J053509.77-052326.9 & V1326 Ori & $-13.23 \pm 5.17$ & $-0.54 \pm 1.96$ & $-14.3 \pm 5.17$ & $0.3 \pm 1.97$ \\
\hline J053510.65-052303.4 & COUP 391 & $0.59 \pm 1.48$ & $6.0 \pm 3.08$ & $-0.48 \pm 1.48$ & $6.84 \pm 3.08$ \\
\hline J053510.73-052344.6 & COUP 394 & $-0.46 \pm 0.93$ & $1.96 \pm 0.97$ & $-1.53 \pm 0.93$ & $2.8 \pm 0.98$ \\
\hline J053511.74-052351.6 & COUP 443 & $4.92 \pm 0.97$ & $2.69 \pm 1.63$ & $3.85 \pm 0.97$ & $3.53 \pm 1.64$ \\
\hline J053511.80-052149.2 & GMR A & $1.63 \pm 0.89$ & $-2.69 \pm 1.28$ & $0.56 \pm 0.89$ & $-1.85 \pm 1.29$ \\
\hline J053512.71-052353.1 & $\cdots$ & $1.06 \pm 2.47$ & $-0.02 \pm 4.3$ & $-0.01 \pm 2.47$ & $0.82 \pm 4.3$ \\
\hline J053512.78-052410.7 & COUP 496 & $0.11 \pm 1.64$ & $1.83 \pm 2.4$ & $-0.96 \pm 1.64$ & $2.67 \pm 2.41$ \\
\hline J053512.97-052330.0 & COUP 509 & $-1.09 \pm 2.59$ & $-4.1 \pm 1.81$ & $-2.16 \pm 2.59$ & $-3.26 \pm 1.82$ \\
\hline J053512.98-052355.0 & COUP 510 & $1.93 \pm 1.46$ & $-4.2 \pm 5.64$ & $0.86 \pm 1.46$ & $-3.36 \pm 5.64$ \\
\hline J053513.11-052247.3 & [B2000] j131-247 & $-0.37 \pm 2.02$ & $-0.7 \pm 1.59$ & $-1.44 \pm 2.02$ & $0.14 \pm 1.6$ \\
\hline J053513.21-052254.8 & COUP 530 & $-1.08 \pm 0.65$ & $-0.38 \pm 1.54$ & $-2.15 \pm 0.66$ & $0.46 \pm 1.55$ \\
\hline J053513.41-052411.2 & Zapata 10 & $0.17 \pm 0.88$ & $-0.95 \pm 2.11$ & $-0.9 \pm 0.88$ & $-0.11 \pm 2.12$ \\
\hline J053513.59-052355.3 & COUP 554 & $0.69 \pm 1.03$ & $2.01 \pm 1.16$ & $-0.38 \pm 1.03$ & $2.85 \pm 1.17$ \\
\hline J053513.93-052320.1 & COUP 593 & $3.64 \pm 1.47$ & $0.34 \pm 0.78$ & $2.57 \pm 1.47$ & $1.18 \pm 0.8$ \\
\hline J053513.97-052409.8 & COUP 594 & $0.75 \pm 0.59$ & $1.49 \pm 0.66$ & $-0.32 \pm 0.6$ & $2.33 \pm 0.68$ \\
\hline J053514.03-052223.2 & Zapata 11 & $-10.68 \pm 5.59$ & $8.43 \pm 2.33$ & $-11.75 \pm 5.59$ & $9.27 \pm 2.34$ \\
\hline J053514.14-052356.8 & COUP 607 & $3.0 \pm 2.02$ & $1.56 \pm 3.12$ & $1.93 \pm 2.02$ & $2.4 \pm 3.12$ \\
\hline J053514.16-052301.1 & GMR C & $1.36 \pm 0.5$ & $-0.88 \pm 0.92$ & $0.29 \pm 0.51$ & $-0.04 \pm 0.93$ \\
\hline J053514.33-052317.4 & COUP 625 & $2.28 \pm 0.87$ & $1.2 \pm 1.23$ & $1.21 \pm 0.87$ & $2.04 \pm 1.24$ \\
\hline J053514.50-052238.7 & COUP 639 & $1.18 \pm 1.06$ & $-0.91 \pm 0.99$ & $0.11 \pm 1.06$ & $-0.07 \pm 1.0$ \\
\hline J053514.55-052316.0 & COUP 640 & $0.36 \pm 2.09$ & $-7.86 \pm 6.72$ & $-0.71 \pm 2.09$ & $-7.02 \pm 6.72$ \\
\hline J053514.61-052221.0 & IRc 23 & $4.43 \pm 3.72$ & $19.29 \pm 8.64$ & $3.36 \pm 3.72$ & $20.13 \pm 8.64$ \\
\hline J053514.65-052233.7 & Parenago 1839 & $-6.63 \pm 7.1$ & $-2.37 \pm 2.39$ & $-7.7 \pm 7.1$ & $-1.53 \pm 2.4$ \\
\hline J053514.66-052211.2 & COUP 647 & $-2.09 \pm 2.97$ & $-0.9 \pm 1.37$ & $-3.16 \pm 2.97$ & $-0.06 \pm 1.38$ \\
\hline J053514.69-052211.0 & COUP 647 & $0.26 \pm 1.34$ & $-2.14 \pm 1.42$ & $-0.81 \pm 1.34$ & $-1.3 \pm 1.43$ \\
\hline J053514.81-052304.8 & MLLA 472 & $3.24 \pm 0.73$ & $-0.43 \pm 1.28$ & $2.17 \pm 0.74$ & $0.41 \pm 1.29$ \\
\hline J053514.90-052225.4 & GMR D & $-0.05 \pm 1.07$ & $-2.44 \pm 0.82$ & $-1.12 \pm 1.07$ & $-1.6 \pm 0.84$ \\
\hline J053514.93-052329.0 & COUP 671 & $1.44 \pm 1.68$ & $2.67 \pm 1.72$ & $0.37 \pm 1.68$ & $3.51 \pm 1.73$ \\
\hline J053514.95-052339.2 & Parenago 1844 & $-0.54 \pm 1.96$ & $-0.08 \pm 2.16$ & $-1.61 \pm 1.96$ & $0.76 \pm 2.17$ \\
\hline J053515.03- 052231.1 & MLLA 606 & $-1.77 \pm 8.47$ & $-12.23 \pm 3.75$ & $-2.84 \pm 8.47$ & $-11.39 \pm 3.75$ \\
\hline J053515.21-052318.8 & COUP 690 & $2.84 \pm 1.1$ & $2.38 \pm 1.18$ & $1.77 \pm 1.1$ & $3.22 \pm 1.19$ \\
\hline J053515.26-052256.9 & Zapata 29 & $1.97 \pm 0.91$ & $-0.81 \pm 0.98$ & $0.9 \pm 0.91$ & $0.03 \pm 0.99$ \\
\hline J053515.36-052321.4 & MLLA 410 & $2.58 \pm 0.93$ & $-0.88 \pm 0.92$ & $1.51 \pm 0.93$ & $-0.04 \pm 0.93$ \\
\hline J053515.36-052324.1 & MLLA 391 & $0.19 \pm 1.0$ & $-2.25 \pm 1.37$ & $-0.88 \pm 1.0$ & $-1.41 \pm 1.38$ \\
\hline J053515.38-052225.4 & MLLA 630C & $0.75 \pm 1.24$ & $-9.28 \pm 3.88$ & $-0.32 \pm 1.24$ & $-8.44 \pm 3.88$ \\
\hline J053515.40-052240.0 & [H97b] 20055 & $2.31 \pm 1.01$ & $0.49 \pm 1.2$ & $1.24 \pm 1.01$ & $1.33 \pm 1.21$ \\
\hline J053515.44-052345.5 & Parenago 1868 & $3.04 \pm 0.84$ & $-1.49 \pm 0.88$ & $1.97 \pm 0.84$ & $-0.65 \pm 0.89$ \\
\hline J053515.52-052337.4 & GMR 14 & $2.29 \pm 0.76$ & $0.97 \pm 0.72$ & $1.22 \pm 0.77$ & $1.81 \pm 0.74$ \\
\hline J053515.73-052322.5 & GMR 26 & $2.75 \pm 0.44$ & $-0.87 \pm 0.31$ & $1.68 \pm 0.45$ & $-0.03 \pm 0.35$ \\
\hline J053515.77-052309.9 & $\theta^{1}$ Ori E & $1.67 \pm 0.39$ & $0.8 \pm 0.58$ & $0.6 \pm 0.4$ & $1.64 \pm 0.6$ \\
\hline J053515.80-052326.5 & GMR 13 & $1.78 \pm 0.34$ & $1.17 \pm 0.5$ & $0.71 \pm 0.35$ & $2.01 \pm 0.52$ \\
\hline J053515.83-052314.1 & GMR 12 & $4.34 \pm 0.22$ & $-1.94 \pm 0.28$ & $3.27 \pm 0.24$ & $-1.1 \pm 0.32$ \\
\hline J053515.84-052322.5 & GMR 11 & $2.6 \pm 0.27$ & $-0.45 \pm 0.42$ & $1.53 \pm 0.28$ & $0.39 \pm 0.45$ \\
\hline J053515.85-052325.6 & GMR 10 & $2.0 \pm 0.29$ & $-2.76 \pm 0.42$ & $0.93 \pm 0.3$ & $-1.92 \pm 0.45$ \\
\hline J053515.88-052301.9 & COUP 743 & $-0.65 \pm 1.64$ & $-2.14 \pm 1.61$ & $-1.72 \pm 1.64$ & $-1.3 \pm 1.62$ \\
\hline J053515.91-052338.0 & GMR 24 & $3.78 \pm 0.59$ & $-1.09 \pm 0.78$ & $2.71 \pm 0.6$ & $-0.25 \pm 0.8$ \\
\hline J053515.91-052417.8 & COUP 748 & $2.52 \pm 1.89$ & $-0.05 \pm 1.25$ & $1.45 \pm 1.89$ & $0.79 \pm 1.26$ \\
\hline J053515.95-052349.8 & GMR 9 & $0.6 \pm 0.4$ & $-2.1 \pm 1.05$ & $-0.47 \pm 0.41$ & $-1.26 \pm 1.06$ \\
\hline J053516.00-052353.0 & Zapata 46 & $3.67 \pm 1.56$ & $-0.15 \pm 1.22$ & $2.6 \pm 1.56$ & $0.69 \pm 1.23$ \\
\hline J053516.07- 052324.4 & GMR 8 & $2.04 \pm 0.33$ & $-2.97 \pm 0.37$ & $0.97 \pm 0.34$ & $-2.13 \pm 0.4$ \\
\hline J053516.07-052307.0 & GMR 15 & $0.54 \pm 0.27$ & $1.35 \pm 0.6$ & $-0.53 \pm 0.28$ & $2.19 \pm 0.62$ \\
\hline J053516.08-052327.8 & GMR 22 & $2.56 \pm 0.57$ & $1.07 \pm 0.33$ & $1.49 \pm 0.58$ & $1.91 \pm 0.37$ \\
\hline J053516.10-052323.0 & TCC 58 & $3.2 \pm 0.94$ & $-1.76 \pm 1.69$ & $2.13 \pm 0.94$ & $-0.92 \pm 1.7$ \\
\hline J053516.11-052314.3 & TCC 59 & $2.39 \pm 0.93$ & $-0.47 \pm 0.96$ & $1.32 \pm 0.93$ & $0.37 \pm 0.97$ \\
\hline J053516.29-052316.6 & GMR 7 & $0.82 \pm 0.23$ & $0.24 \pm 0.3$ & $-0.25 \pm 0.25$ & $1.08 \pm 0.34$ \\
\hline J053516.33-052322.6 & GMR 16 & $-1.56 \pm 0.36$ & $1.54 \pm 0.2$ & $-2.63 \pm 0.37$ & $2.38 \pm 0.26$ \\
\hline J053516.34-052249.0 & Zapata 54 & $3.78 \pm 0.78$ & $-2.72 \pm 1.01$ & $2.71 \pm 0.79$ & $-1.88 \pm 1.02$ \\
\hline J053516.38-052403.3 & Parenago 1895 & $3.25 \pm 1.86$ & $-0.99 \pm 1.49$ & $2.18 \pm 1.86$ & $-0.15 \pm 1.5$ \\
\hline J053516.40-052235.2 & GMR K & $-2.51 \pm 0.89$ & $1.24 \pm 1.5$ & $-3.58 \pm 0.89$ & $2.08 \pm 1.51$ \\
\hline J053516.47-052322.9 & $\theta^{1}$ Ori C & $1.68 \pm 1.4$ & $-3.01 \pm 1.94$ & $0.61 \pm 1.4$ & $-2.17 \pm 1.95$ \\
\hline J053516.59-052250.3 & MLLA 532 & $0.76 \pm 1.68$ & $-2.34 \pm 1.39$ & $-0.31 \pm 1.68$ & $-1.5 \pm 1.4$ \\
\hline
\end{tabular}


Table 2

(Continued)

\begin{tabular}{|c|c|c|c|c|c|}
\hline \multirow[b]{2}{*}{ VLA Name } & \multirow[b]{2}{*}{$\begin{array}{l}\text { Other } \\
\text { Name }^{\mathrm{a}}\end{array}$} & \multicolumn{2}{|c|}{ Absolute } & \multicolumn{2}{|c|}{ Orion's Rest Frame } \\
\hline & & $\begin{array}{c}\mu_{\alpha} \cos \delta \\
\left(\mathrm{mas} \mathrm{yr}^{-1}\right)\end{array}$ & $\begin{array}{c}\mu_{\delta} \\
\left(\operatorname{mas~yr}^{-1}\right)\end{array}$ & $\begin{array}{c}\mu_{\alpha} \cos \delta \\
\left(\mathrm{mas} \mathrm{yr}^{-1}\right)\end{array}$ & $\begin{array}{c}\mu_{\delta} \\
\left(\operatorname{mas~yr}^{-1}\right)\end{array}$ \\
\hline J053516.62-052316.1 & GMR 21 & $1.32 \pm 0.67$ & $-3.08 \pm 0.61$ & $0.25 \pm 0.68$ & $-2.24 \pm 0.63$ \\
\hline J053516.75-052316.5 & GMR 6 & $1.19 \pm 0.34$ & $-1.64 \pm 0.47$ & $0.12 \pm 0.35$ & $-0.8 \pm 0.5$ \\
\hline J053516.77-052404.3 & V1279 Ori & $0.13 \pm 0.81$ & $-1.66 \pm 2.47$ & $-0.94 \pm 0.82$ & $-0.82 \pm 2.48$ \\
\hline J053516.77-052328.1 & GMR 17 & $0.24 \pm 0.44$ & $-3.14 \pm 0.35$ & $-0.83 \pm 0.45$ & $-2.3 \pm 0.38$ \\
\hline J053516.85-052326.2 & GMR 5 & $-1.11 \pm 0.37$ & $-1.76 \pm 0.43$ & $-2.18 \pm 0.38$ & $-0.92 \pm 0.46$ \\
\hline J053516.89-052338.1 & Zapata 62 & $1.98 \pm 1.83$ & $-2.05 \pm 1.71$ & $0.91 \pm 1.83$ & $-1.21 \pm 1.72$ \\
\hline J053516.97-052248.7 & GMR E & $-0.66 \pm 0.66$ & $-0.98 \pm 0.5$ & $-1.73 \pm 0.67$ & $-0.14 \pm 0.52$ \\
\hline J053516.98-052337.0 & GMR 4 & $2.15 \pm 0.46$ & $0.41 \pm 0.79$ & $1.08 \pm 0.47$ & $1.25 \pm 0.81$ \\
\hline J053516.98- 052300.9 & COUP 845 & $4.2 \pm 1.23$ & $2.26 \pm 0.87$ & $3.13 \pm 1.23$ & $3.1 \pm 0.88$ \\
\hline J053517.01-052233.0 & V1333 Ori & $0.53 \pm 2.84$ & $-3.01 \pm 2.58$ & $-0.54 \pm 2.84$ & $-2.17 \pm 2.58$ \\
\hline J053517.07-052334.0 & GMR 3 & $0.99 \pm 0.49$ & $1.43 \pm 0.33$ & $-0.08 \pm 0.5$ & $2.27 \pm 0.37$ \\
\hline J053517.33-052341.4 & [H97b] 20009 & $3.76 \pm 0.69$ & $0.04 \pm 1.3$ & $2.69 \pm 0.7$ & $0.88 \pm 1.31$ \\
\hline J053517.35-052235.9 & GMR L & $1.12 \pm 0.39$ & $0.29 \pm 1.02$ & $0.05 \pm 0.4$ & $1.13 \pm 1.03$ \\
\hline J053517.39-052203.6 & MLLA 712 & $-3.81 \pm 1.88$ & $-3.98 \pm 2.39$ & $-4.88 \pm 1.88$ & $-3.14 \pm 2.4$ \\
\hline J053517.47-052321.1 & COUP 885 & $0.42 \pm 1.06$ & $2.29 \pm 1.02$ & $-0.65 \pm 1.06$ & $3.13 \pm 1.03$ \\
\hline J053517.48-052251.2 & [H97b] 20031 & $2.0 \pm 0.89$ & $2.32 \pm 1.61$ & $0.93 \pm 0.89$ & $3.16 \pm 1.62$ \\
\hline J053517.56-052324.9 & GMR 2 & $0.65 \pm 0.4$ & $0.27 \pm 0.38$ & $-0.42 \pm 0.41$ & $1.11 \pm 0.41$ \\
\hline J053517.68-052340.9 & GMR 1 & $0.91 \pm 0.67$ & $-0.34 \pm 0.72$ & $-0.16 \pm 0.68$ & $0.5 \pm 0.74$ \\
\hline J053517.95-052245.4 & GMR G & $2.0 \pm 0.58$ & $1.73 \pm 0.79$ & $0.93 \pm 0.59$ & $2.57 \pm 0.81$ \\
\hline J053518.05-052330.7 & GMR 19 & $1.26 \pm 0.58$ & $-1.0 \pm 0.7$ & $0.19 \pm 0.59$ & $-0.16 \pm 0.72$ \\
\hline J053518.24-052315.6 & Zapata 75 & $1.59 \pm 0.74$ & $0.31 \pm 0.46$ & $0.52 \pm 0.75$ & $1.15 \pm 0.49$ \\
\hline J053518.37-052237.4 & GMR F & $2.12 \pm 0.72$ & $1.07 \pm 0.63$ & $1.05 \pm 0.73$ & $1.91 \pm 0.65$ \\
\hline J053518.86-052141.2 & Parenago 1924 & $-2.26 \pm 2.84$ & $-1.16 \pm 1.84$ & $-3.33 \pm 2.84$ & $-0.32 \pm 1.85$ \\
\hline J053520.15-052228.2 & COUP 1084 & $3.6 \pm 1.6$ & $2.46 \pm 1.45$ & $2.53 \pm 1.6$ & $3.3 \pm 1.46$ \\
\hline J053520.72-052144.3 & V1239 Ori & $3.15 \pm 3.59$ & $3.54 \pm 4.09$ & $2.08 \pm 3.59$ & $4.38 \pm 4.09$ \\
\hline
\end{tabular}

Note.

${ }^{a}$ COUP—Getman et al. (2005), V—Kukarkin et al. (1971), GMR—Garay et al. (1987), [B2000]—Bally et al. (2000), Zapata—Zapata et al. (2004), IRc—Rieke et al. (1973), Parenago—Parenago (1954), MLLA—Muench et al. (2002), and [H97b]—Hillenbrand (1997).

GMR 12, GMR G and GMR F) and obtained accurate astrometry for them. Our VLA astrometry for these sources is consistent to better than $1.5 \times \sigma$ with that reported by Menten et al. (2007). The only exception is for the R.A. component of the proper motion of GMR G, for which the VLBA value is about twice the figure obtained with the VLA. Many VLBAdetected YSOs belong to tight multiple systems (Ortiz-León et al. 2016). In some systems a close companion could cause differences of measured proper motions between the VLA and the VLBA, since the former will measure the total motion of the system and the latter could be dominated by an orbital motion component (e.g., Loinard et al. 2007). More recent VLBA observations of GMR G by Kounkel et al. (2016a) obtained different values for the proper motions than those reported by Menten et al. (2007) suggesting that the motion of the source is not uniform, perhaps due to a close companion.

\section{ANALYSIS AND DISCUSSION}

\subsection{Mean Proper Motions and Velocity Dispersions}

From the measured proper motion we obtained the mean velocity along both axes (R.A. and decl.). This information is then used to identify stars with large peculiar motions. As is shown in Figure 3, stars with proper motions three or more times above the mean proper motion values (red squares) are subsequently not used for our internal kinematic analysis and are discussed separately. These stars are also shown as red arrows in Figure 1.
For the remaining stars the mean proper motions are:

$$
\begin{aligned}
\overline{\mu_{\alpha} \cos \delta} & =1.07 \pm 0.09 \operatorname{mas~yr}^{-1} \\
\overline{\mu_{\delta}} & =-0.84 \pm 0.16 \operatorname{mas~yr}^{-1}
\end{aligned}
$$

Compared with those obtained by Gómez et al. (2005), the mean proper motion in R.A. agrees within errors. On the other hand, the decl. value does not. As discussed in the previous section, the results of Gómez et al. (2005) were affected by a systematic shift in this coordinate for the 1991.68 epoch (Gómez et al. 2008). We attribute the differences to this shift. We argue that the values quoted above represent the bulk relative motion between the ONC and the Sun.

The transformation of the mean proper motions to the Galactic coordinate system yields

$$
\begin{aligned}
\overline{\mu_{l} \cos b} & =1.22 \pm 0.17 \mathrm{mas} \mathrm{yr}^{-1} \\
\overline{\mu_{b}} & =0.58 \pm 0.11 \mathrm{mas} \mathrm{yr}^{-1}
\end{aligned}
$$

The theoretical values of proper motions expected for a source in Orion, can be derived from a Galactic rotation model and compared with our results. To estimate this expected motion, we assume, first, that the stars move in circular orbits around the Galactic center with an LSR speed of $254 \mathrm{~km} \mathrm{~s}^{-1}$ (Reid et al. 2009). Second, we adopt a solar motion relative to the LSR of $(U, V, W)=(11.10,12.24,7.25) \mathrm{km} \mathrm{s}^{-1}$ (Schönrich et al. 2010). Finally, we assume that the Sun is at a distance of $8.4 \mathrm{kpc}$ from the Galactic center (Reid et al. 2009). From this 


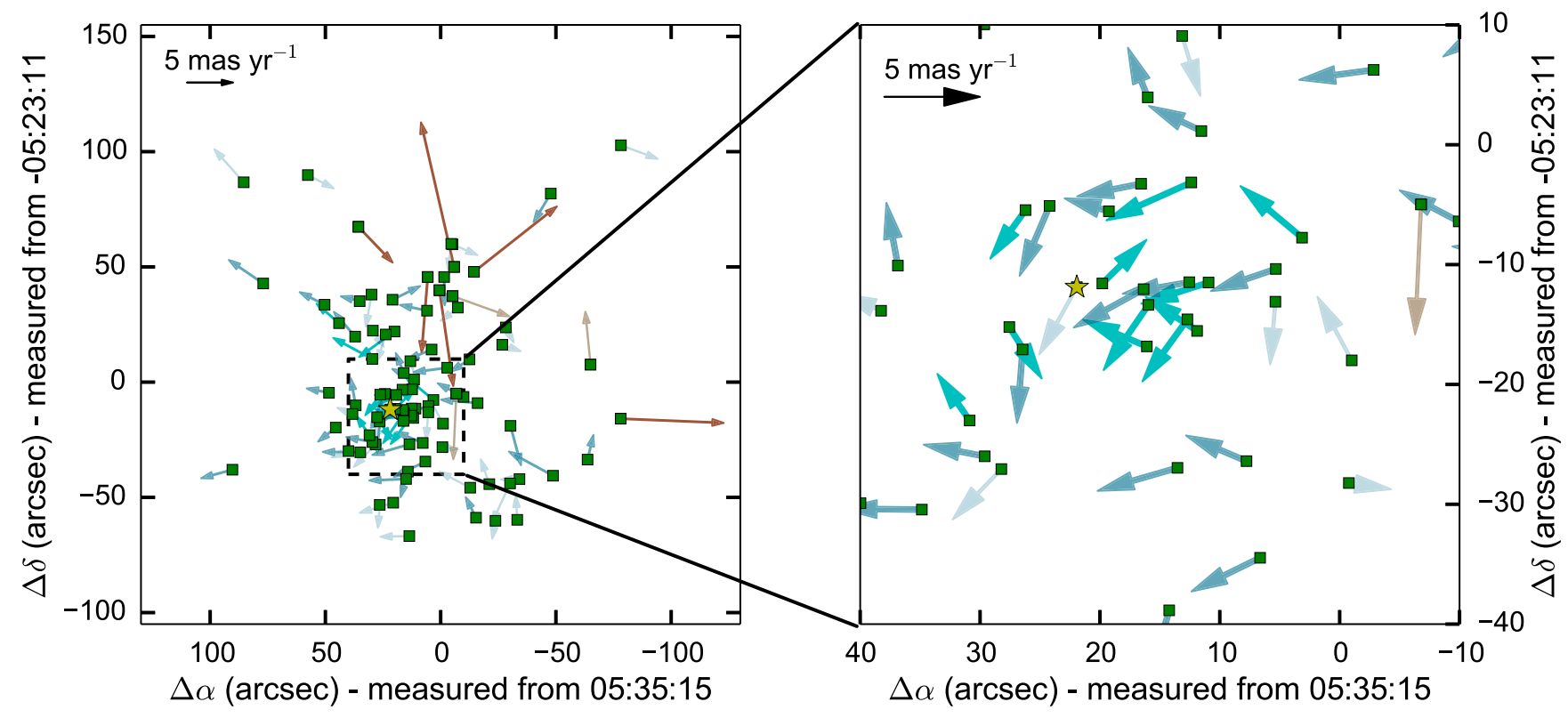

Figure 1. Absolute proper motions of radio stellar sources as distributed on the plane of the sky. Cyan arrows are proper motions that are within 3 sigma of the mean proper motions (see text) and red arrows correspond to those sources above this limit. The yellow star indicates the position of the massive star $\theta^{1}$ Ori C. The level of transparency of the arrows indicates the significance of the measured proper motions. Left: the complete sample of measured proper motions. Right: a zoom to the central region.

Galactic rotation model the expected Galactic proper motions for stars at the position of Orion are:

$$
\begin{aligned}
\overline{\mu_{l} \cos b}(\exp ) & =1.39 \pm 0.05 \mathrm{mas} \mathrm{yr}^{-1}, \\
\overline{\mu_{b}}(\exp ) & =-0.02 \pm 0.02 \mathrm{mas} \mathrm{yr}^{-1} .
\end{aligned}
$$

Thus, the average proper motion in Galactic longitude of Orion is consistent with those expected from models of Galactic rotation. In Galactic latitude there is a peculiar motion of $0.60 \pm 0.12 \mathrm{mas} \mathrm{yr}^{-1} \equiv 1.18 \pm 0.24 \mathrm{~km} \mathrm{~s}^{-1}$ toward the Galactic plane.

Having measured the mean proper motion of the central ONC, we subtracted it from the individual measured proper motions to calculate the proper motions of the sources in the rest frame of the ONC (Figure 4). The dispersion $(\sigma)$ of these proper motions (after correction for the errors of measurement following Jones \& Walker 1988) are:

$$
\begin{aligned}
\sigma_{\alpha} & =1.08 \pm 0.07 \mathrm{mas} \mathrm{yr}^{-1} \equiv 2.12 \pm 0.13 \mathrm{~km} \mathrm{~s}^{-1}, \\
\sigma_{\delta} & =1.27 \pm 0.15 \mathrm{mas} \mathrm{yr}^{-1} \equiv 2.49 \pm 0.29 \mathrm{~km} \mathrm{~s}^{-1} .
\end{aligned}
$$

The velocity dispersion values are lower than those found by Gómez et al. (2005) for their radio sources analysis

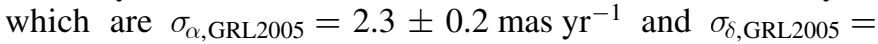
$3.1 \pm 0.2 \mathrm{mas} \mathrm{yr}^{-1}$. The differences in the results can be attributed to the different number of analyzed sources and to the fact that Gómez et al. (2005) did not correct for the errors of measurement. Our measured velocity dispersions are in agreement with the velocity dispersions found by Jones \& Walker (1988) from optical observation of $\sigma_{\alpha, \mathrm{JW} 1998}=$ $0.91 \pm 0.06 \mathrm{mas} \mathrm{yr}^{-1}$ and $\sigma_{\delta, \mathrm{JW} 1998}=1.18 \pm 0.05 \mathrm{mas} \mathrm{yr}^{-1}$. Our computed velocity dispersions are also similar to the stellar radial velocity dispersion in the $\mathrm{ONC}$ of $\sigma_{V_{\text {rad }}} \simeq 2.5$ $\mathrm{km} \mathrm{s}^{-1}$ (Kounkel et al. 2016b). Finally, they are also similar to the velocity dispersion on the plane of the sky of the ONC ionized gas of $\sigma_{\mathrm{pos}} \simeq 3 \pm 1 \mathrm{~km} \mathrm{~s}^{-1}$, but significantly lower than the velocity dispersion along the line of sight of the ONC ionized gas $\sigma_{\mathrm{los}} \simeq 6 \pm 1 \mathrm{~km} \mathrm{~s}^{-1}$ (Arthur et al. 2016). However, the latter could be affected by large velocity gradients and emissivity fluctuations along the line of sight (Arthur et al. 2016).

\subsection{The Internal Kinematics}

According to the review of Muench et al. (2008, p. 483), there is some conflict between results of studies of the kinematics of stars in Orion. Some authors (e.g., Parenago 1954; Strand 1958; Fallon et al. 1977) have claimed evidence of expansion or contraction, but it has also been claimed that these results are due to observational errors (e.g., Vasilevskis 1962, 1971; Allen et al. 1974). Furthermore, given the velocity dispersions, one would expect the ONC core to be virialized and not contracting nor expanding motions are expected (e.g., Hillenbrand \& Hartmann 1998).

Using our measured proper motions, we searched for evidence of organized motions, specifically for expansion (or contraction) and rotation. First, we will use the proper motions of the radio sources relative to the Orion rest frame that are listed in columns 5 and 6 of Table 2. We follow Rivera et al. (2015) and define the vectors $r_{*}, \hat{r}_{*}$ and $\delta v_{*}$ for the position, the unit vector associated to the position, and the velocity for each star with respect to the center of the group. The cross products $\hat{r}_{*} \times \delta v_{*}$ and the dot products $\hat{r}_{*} \cdot \delta v_{*}$ are calculated individually for all the stars. The mean values of the cross and dot products can be used to search for organized movement. In a purely radial movement the mean cross product is expected to be zero, while the mean dot product will be large (positive for expansion and negative for contraction). On the other hand, for pure rotation the mean cross product will be large and the mean dot product is zero. For the YSOs in Orion we obtained:

$$
\begin{aligned}
\overline{\hat{r}_{*} \times \delta v_{*}} & =0.7 \pm 0.3 \mathrm{~km} \mathrm{~s}^{-1}, \quad \text { and } \\
\overline{\hat{r}_{*} \cdot \delta v_{*}} & =-0.1 \pm 0.3 \mathrm{~km} \mathrm{~s}^{-1} \text {. }
\end{aligned}
$$



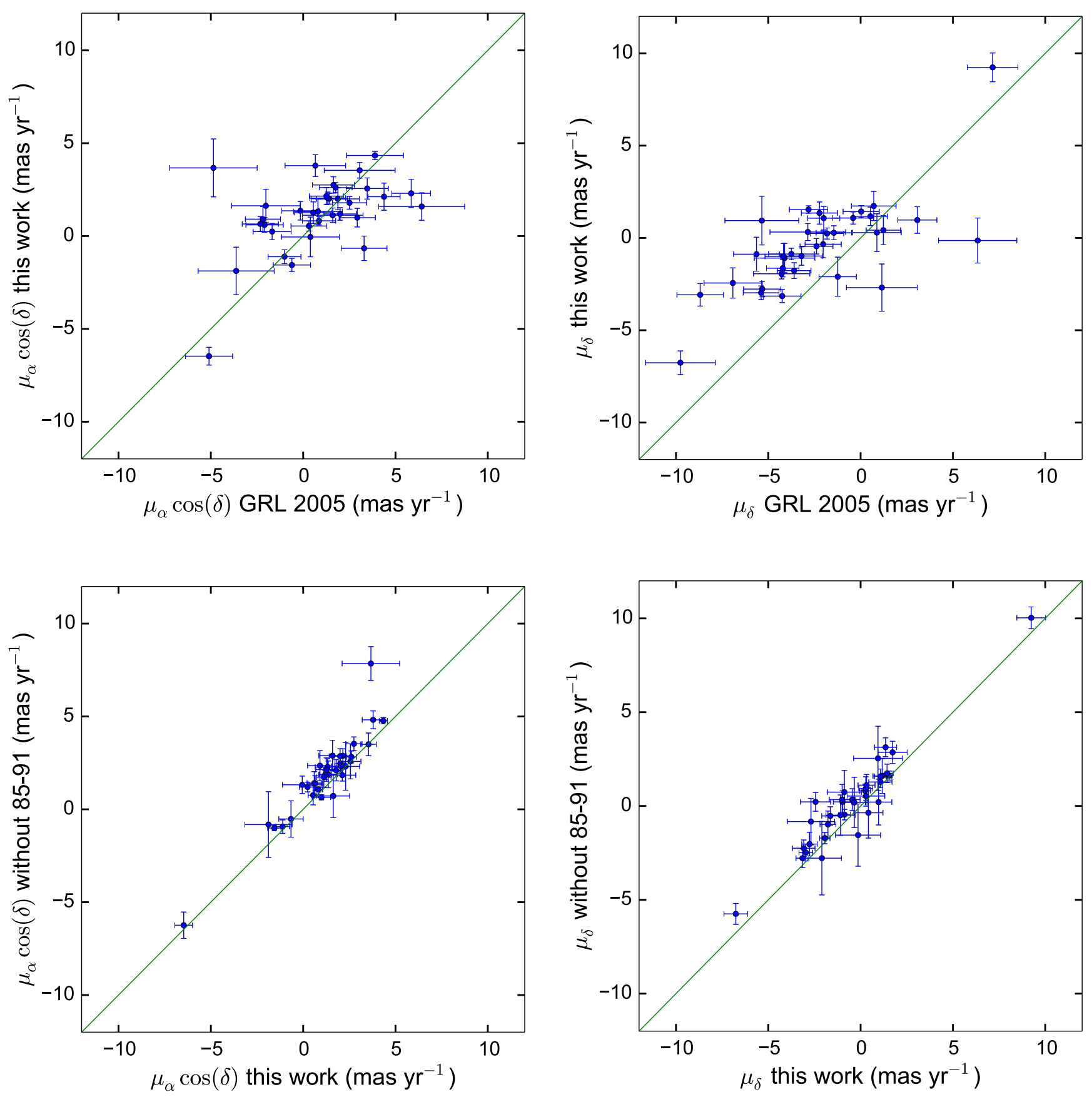

Figure 2. The top panels show our measured proper motions against the proper motions measured by Gómez et al. (2005; GRL 2005). The bottom panels show our measured proper motions with all epochs considered against our measured proper motions without epochs 1985.05 and 1991.68. The left panels are for R.A. and the right panels are for decl.

These numbers are small in comparison with the velocity dispersion, so our results do not point toward the existence of organized motions in the central ONC. In particular there are no signs of expansion or contraction and this result agrees with previous discussions by Vasilevskis $(1962,1971)$ and Allen et al. (1974).

\subsection{Fast Moving Sources}

Stars with large peculiar velocities have been reported in the Orion Trapezium-BN/KL region in the past. The more interesting case is found in the $\mathrm{BN} / \mathrm{KL}$ region where three stars (sources BN, I, and the two components related to source $n$ ) were suggested to participate in a dynamical decay event around 550 years ago (Gómez et al. 2005, 2008; Rodríguez et al. 2005, 2017), this event could also be related to the explosive phenomenon in the $\mathrm{BN} / \mathrm{KL}$ region (Bally \& Zinnecker 2005). Two other stars were proposed to be ejected from the Orion Trapezium and one more was proposed to be ejected from the molecular core [TUK93] 28 a few thousand years ago (Poveda et al. 2005). The proposed mechanism that produce these fast moving sources is $n$-body interactions (Poveda et al. 1967). We will use our results to identify new candidates to fast moving sources in this region.

From the 88 radio proper motion reported in this paper, we found that, in addition to the four sources in the Orion $\mathrm{BN} / \mathrm{KL}$ 

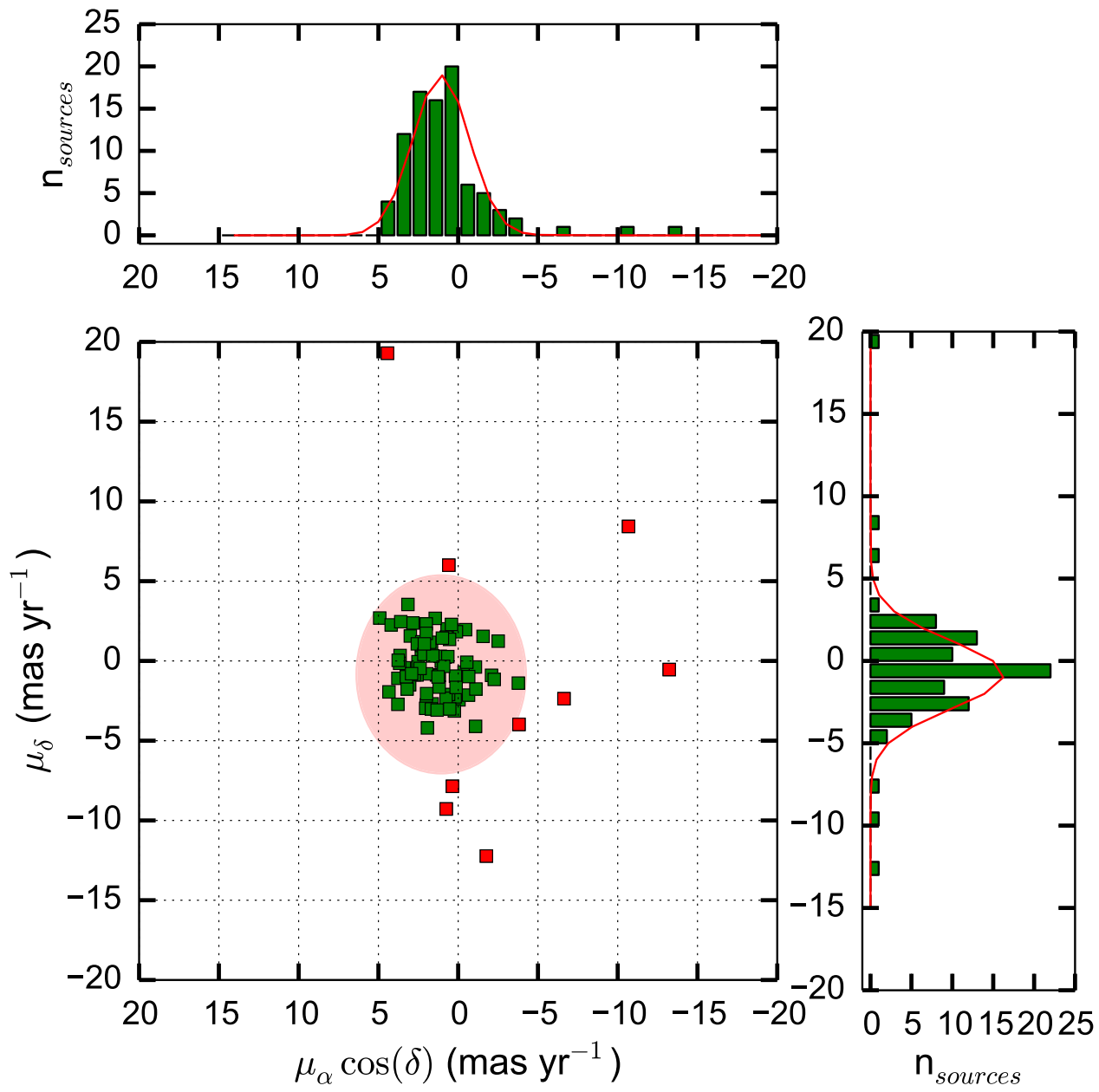

Figure 3. Source distribution in the $\left(\mu_{\alpha} \cos (\delta), \mu_{\delta}\right)$ plane. Top and right panel shows the histogram distribution in R.A. and decl., respectively, together with the Gaussian fits to the data.

region, nine present proper motion significantly different from the rest of the radio sources in the core of the ONC (Figure 1). These sources are: V 1326 Ori, COUP 391, COUP 640, Parenago 1839, MLLA 606, MLLA 630C, MLLA 712, Zapata 11 and IRc 23. The measured proper motions of COUP 640 and Parenago 1839 are below 1- $\sigma$, and thus they are not significant. We now discuss what could be the origin of the peculiar proper motions of the remaining fast moving sources.

First, we check if these sources are foreground objects (e.g., Alves \& Bouy 2012). Sources at closer distances than Orion will have larger proper motions than sources that are actually part of this region. From the Galactic rotation model described above we obtained the expected proper motions of sources in the direction to Orion at different distances (Table 3). From this table we note that stars at closer distances will have larger and more negative proper motion in decl., while in R.A. the proper motion will be small and positive. Comparing these figures with the measured motions of our high peculiar proper motion sources, we conclude that MLLA 606 and MLLA 630C could be sources at a closer distance to the Sun than the Orion system. Due to their large errors, however, it is not possible to favor any distance, and in fact within errors these sources could also be part of the Orion system.

The proper motion vector of V 1326 Ori is one of the largest in our sample. Interestingly, these motions suggest that in the past it was closer to the densest region of the Trapezium group (see Figure 1). Thus, this source could have been ejected around 7000 years ago from the Trapezium maybe also via $n$ body interactions. The proper motions of this source in the rest frame of the ONC correspond to a linear velocity of $28.0 \pm 10.8 \mathrm{~km} \mathrm{~s}^{-1}$.

The source Zapata 11 is $1 . " 5$ southwest from the famous BN source and it was first noticed by Menten \& Reid (1995), who suggested that it is a jetlike extension of this source. The measured proper motion of Zapata 11 are comparable with those of BN (Gómez et al. 2008; Rodríguez et al. 2017; see also Figure 5) and there is no significant evidence that it is moving away rapidly from BN. Thus Zapata 11 could be, instead, a companion of BN, although the separation between the two ( $621 \mathrm{au})$ seems somewhat large for sources in the region (for example see the discussion by Petr et al. 1998). Additional observations could help to distinguish between these two hypotheses. On the other hand, source IRc 23 is located $\sim 7 ! 5$ northeast of BN. It was first reported at radio frequencies by Forbrich et al. (2016) ${ }^{14}$ with a spectral index that suggests nonthermal emission. Extrapolating the proper motions of IRc 23 to 550 years in the past it appears to agree, within the errors, with the position where sources $\mathrm{BN}$, I, and $\mathrm{n}$ were then

\footnotetext{
${ }^{14}$ It was, however, clearly detected before by Menten \& Reid (1995) and by Gómez et al. (2008). These last authors mislabeled it as source D in their Figure 2.
} 


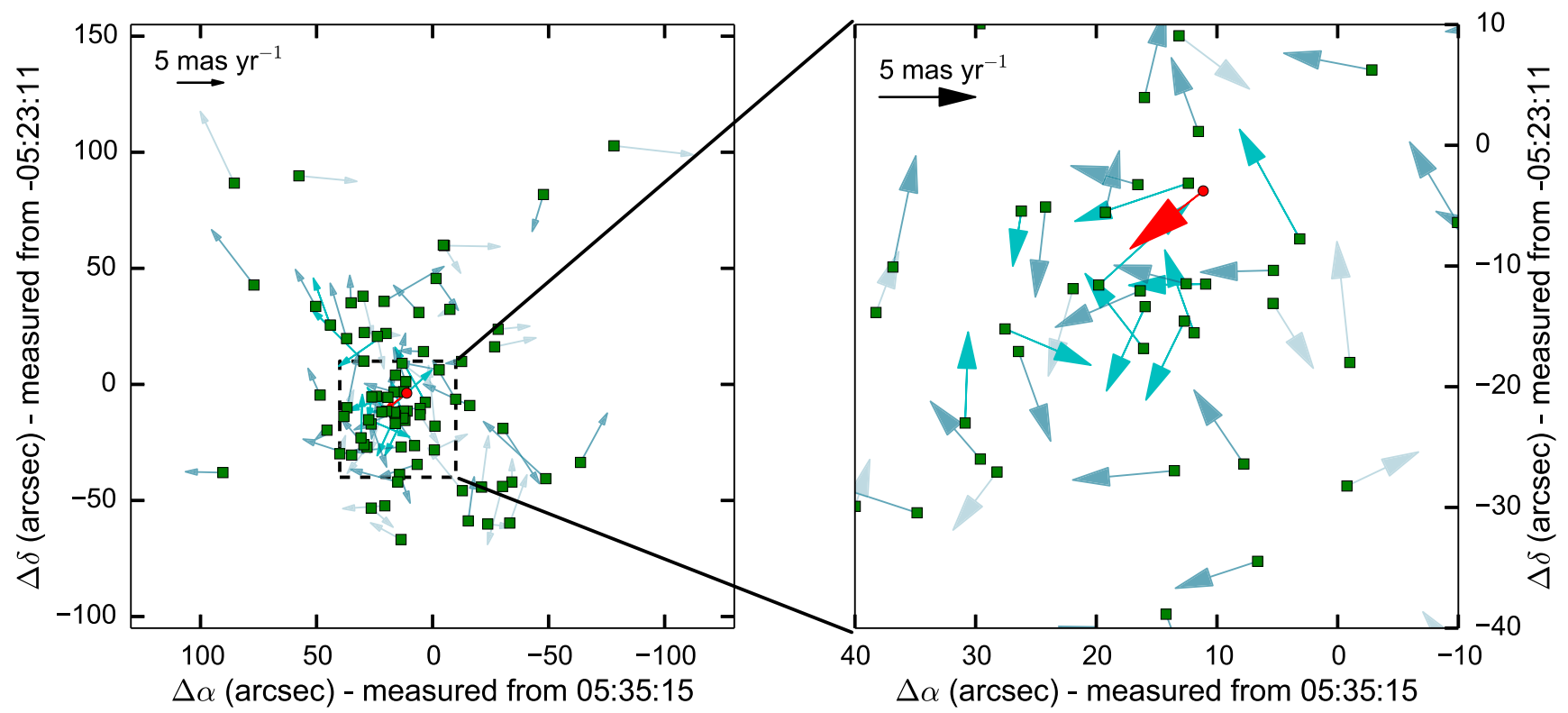

Figure 4. Proper motions relative to the Orion's rest frame of radio stellar sources with normal proper motions. The red arrow is the mean proper motion of the ONC. The level of transparency of the arrows indicates the significance of the measured proper motions. Left: the complete sample of relative proper motions. Right: a zoom to the central region.

Table 3

Expected Proper Motions in the Direction to Orion from Galactic Rotation Model at Different Distances

\begin{tabular}{lcc}
\hline \hline $\begin{array}{l}\text { Distance } \\
(\mathrm{pc})\end{array}$ & $\begin{array}{c}\mu_{\alpha} \\
(\mathrm{mas})\end{array}$ & $\begin{array}{c}\mu_{\delta} \\
(\mathrm{mas})\end{array}$ \\
\hline 100 & $2.10 \pm 2.30$ & $-9.96 \pm 1.17$ \\
200 & $1.11 \pm 0.80$ & $-4.20 \pm 0.40$ \\
300 & $0.79 \pm 0.28$ & $-2.29 \pm 0.15$ \\
400 & $0.63 \pm 0.04$ & $-1.35 \pm 0.04$ \\
\hline
\end{tabular}

(Figure 5). However, due to the large uncertainties, the relation of IRc 23 with the other sources in $\mathrm{BN} / \mathrm{KL}$ needs to be investigated further.

Finally, the sources COUP 391 and MLLA 712 have proper motions with large uncertainties, and within errors they are also consistent within three times the mean proper motion, our criteria to select fast moving sources. Future observations could help to decide if these objects have large peculiar motions or not.

\section{CONCLUSIONS}

Using observations with the VLA radio interferometer that span 29.1 years, we measured the absolute proper motions of 88 YSOs with radio emission in the Orion Trapezium-BN/KL region. The analysis of these proper motions let us reach the following conclusions:

1. The mean proper motions of the Orion Trapezium-BN/ $\mathrm{KL}$ regions are $\overline{\mu_{\alpha} \cos \delta}=1.03 \pm 0.10 \mathrm{mas} \mathrm{yr}^{-1}$ and

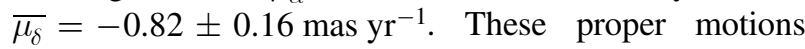
agree within errors with those expected from Galactic rotation curves, with only a small peculiar motion of $1.14 \pm 0.24 \mathrm{~km} \mathrm{~s}^{-1}$ toward the Galactic plane.

2. The calculated velocity dispersions are $\sigma_{\alpha}=$ $2.12 \pm 0.13 \mathrm{~km} \mathrm{~s}^{-1}, \quad \sigma_{\delta}=2.49 \pm 0.29 \mathrm{~km} \mathrm{~s}^{-1}$. These values are in agreement with those obtained at optical wavelengths from proper motions by Jones \& Walker

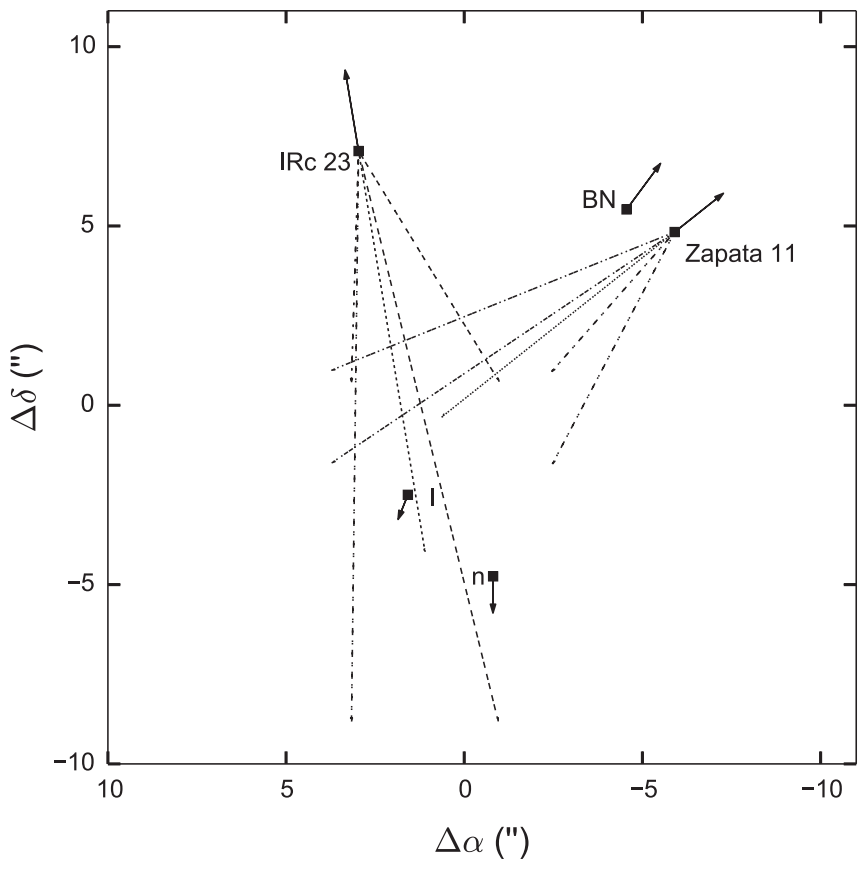

Figure 5. Arrows indicate the direction and proper motion displacement for 100 years, in the rest frame of the Orion radio sources. The dotted and dashed lines indicate the projected movement and errors, respectively, for the last 550 years of sources Zapata 11 and IRc 23. Proper motions of sources BN, I and n were taken from Rodríguez et al. (2017). The origin coordinates are R.A. = $05^{\mathrm{h}} 35^{\mathrm{m}} 14^{\mathrm{s}} .41$; decl. $=-05^{\circ} 22^{\prime} 28^{\prime \prime}$. 1 ; the mean position of sources BN, I and $\mathrm{n}$ on their closest approach around 550 years ago (Rodríguez et al. 2017).

(1988), stellar radial velocities by Kounkel et al. (2016b), and from gas radial velocities by Arthur et al. (2016).

3 . The kinematics of the sources do not show evidence for expansion, contraction, or rotation of the ONC.

4. The large proper motions measured for V 1326 Ori indicate that it could be a runaway star, and the proper motion vector suggests that it might have been ejected from the Orion Trapezium around 7000 years ago. 
5. Extrapolating the proper motions of radio sources Zapata 11 and IRc 23 to 550 years in the past, we find that their positions are close to the intersecting position of sources $\mathrm{BN}$, I and $\mathrm{n}$, the massive stars that participated in a dynamical disintegration. Source Zapata 11 could be either a companion or a slow ejecta of BN.

6. The proper motions of six other radio sources also indicate large peculiar motions. However, within the errors they could also follow the bulk kinematics of the other sources in the region. For two of them, however, the proper motions could be more naturally explained if they were objects along the line of sight to, but at a smaller distance than, the ONC.

The astrometric radio studies of the Orion Trapezium-BN/ KL region appears promising for the future, since Forbrich et al. (2016) detected a total of 556 compact radio sources in this region. With additional new high resolution and high sensitivity radio observations distributed in the next decade, it will be possible to measure the proper motion of these hundreds of sources and perform a comprehensive internal kinematic study of YSOs in the densest region of Orion.

L.L., L.F.R., and J.L.R. acknowledge the financial support of DGAPA, UNAM, and CONACyT, México. The National Radio Astronomy Observatory is operated by Associated Universities Inc. under cooperative agreement with the National Science Foundation. This research has made use of the SIMBAD database, operated at CDS, Strasbourg, France. The Long Baseline Observatory is operated by Associated Universities Inc. under cooperative agreement with the National Science Foundation.

\section{APPENDIX \\ IMPROVEMENT OF THE ERRORS IN PROPER MOTION DETERMINATIONS AS A FUNCTION OF TIME}

The least-squares fitting to the positions of a source as a function of time is a linear regression problem. Following Press et al. (1992), the position $x$ in either R.A. or decl. is described as a function of time $t$ as:

$$
x=a+b t,
$$

where $a$ is the intercept of the line with the $x$-axis and $b$ is the slope. If we have a set of $N$ data points $\left(t_{i}, x_{i}\right)$ we can define the following sums:

$$
\begin{gathered}
S=\sum_{i=0}^{N-1} \frac{1}{\sigma_{i}^{2}} \quad S_{t}=\sum_{i=0}^{N-1} \frac{t_{i}}{\sigma_{i}^{2}} \quad S_{x}=\sum_{i=0}^{N-1} \frac{x_{i}}{\sigma_{i}^{2}} \\
S_{t t}=\sum_{i=0}^{N-1} \frac{t_{i}^{2}}{\sigma_{i}^{2}} \quad S_{t x}=\sum_{i=0}^{N-1} \frac{t_{i} x_{i}}{\sigma_{i}^{2}} .
\end{gathered}
$$

In these sums $\sigma_{i}$ is the error associated to each $x_{i}$ value. Additionally defining

$$
\Delta=S S_{t t}-\left(S_{t}\right)^{2},
$$

the best fit model parameters are given by:

$$
a=\frac{S_{t t} S_{x}-S_{t} S_{t x}}{\Delta},
$$

$$
b=\frac{S S_{t x}-S_{t} S_{x}}{\Delta} .
$$

Finally, the errors of the parameters are given by:

$$
\begin{aligned}
& \sigma_{a}=\sqrt{\frac{S_{t t}}{\Delta}}, \\
& \sigma_{b}=\sqrt{\frac{S}{\Delta}} .
\end{aligned}
$$

To simplify the calculations we will assume that the measurements are made evenly in time:

$$
t=0, \Delta t, 2 \Delta t, 3 \Delta t, \ldots,(N-1) \Delta t,
$$

where $\Delta t$ is the time interval between measurements. We also assume that the error of each measurement is the same:

$$
\sigma_{i}=\sigma .
$$

With these simplifications, three of the sums listed above are given by:

$$
\begin{gathered}
S=\sum_{i=0}^{N-1} \frac{1}{\sigma_{i}^{2}}=\frac{N}{\sigma^{2}} \\
S_{t}=\sum_{i=0}^{N-1} \frac{t_{i}}{\sigma_{i}^{2}}=\frac{N(N-1)}{2 \sigma^{2}} \Delta t \\
S_{t t}=\sum_{i=0}^{N-1} \frac{t_{i}^{2}}{\sigma_{i}^{2}}=\frac{(2 N-1)(N-1)(N)}{6 \sigma^{2}}(\Delta t)^{2} .
\end{gathered}
$$

In Equations (12) and (13) we have used the formulae for the addition of the natural numbers and the addition of the squares of the natural numbers, respectively. Equation (4) can be approximated by:

$$
\Delta=S S_{t t}-\left(S_{t}\right)^{2}=\frac{N^{2}\left(N^{2}-1\right)}{12 \sigma^{4}}(\Delta t)^{2} \simeq \frac{N^{4}}{12 \sigma^{4}}(\Delta t)^{2},
$$

where we have assumed that $N^{2} \gg 1$. Finally, substituting in Equations (7) and (8) we obtain:

$$
\begin{gathered}
\sigma_{a}=\sqrt{\frac{S_{t t}}{\Delta}}=\sigma \sqrt{\frac{4}{N}}, \\
\sigma_{b}=\sqrt{\frac{S}{\Delta}}=\frac{\sigma}{\Delta t} \sqrt{\frac{12}{N^{3}}} .
\end{gathered}
$$

In summary, the error in the intercept will decrease as time $e^{-1 / 2}$, while the error in the slope will decrease as time $e^{-3 / 2}$. Thus, duplicating the time coverage will improve the determination of proper motions by an important factor of 2.8 .

\section{REFERENCES}

Allen, C., Poveda, A., \& Worley, C. E. 1974, RMxAA, 1, 101

Alves, J., \& Bouy, H. 2012, A\&A, 547, A97

Arthur, S. J., Medina, S.-N. X., \& Henney, W. J. 2016, MNRAS, 463, 2864 Bally, J., O’Dell, C. R., \& McCaughrean, M. J. 2000, AJ, 119, 2919

Bally, J., \& Zinnecker, H. 2005, AJ, 129, 2281

Churchwell, E., Felli, M., Wood, D. O. S., \& Massi, M. 1987, ApJ, 321, 516 Dzib, S. A., Loinard, L., Rodríguez, L. F., \& Galli, P. 2014, ApJ, 788, 162 Fallon, F. W., Gerola, H., \& Sofia, S. 1977, ApJ, 217, 719 Forbrich, J., Rivilla, V. M., Menten, K. M., et al. 2016, ApJ, 822, 93 Garay, G., Moran, J. M., \& Reid, M. J. 1987, ApJ, 314, 535 Getman, K. V., Flaccomio, E., Broos, P. S., et al. 2005, ApJS, 160, 319 Gómez, L., Rodríguez, L. F., Loinard, L., et al. 2005, ApJ, 635, 1166 Gómez, L., Rodríguez, L. F., Loinard, L., et al. 2008, ApJ, 685, 333 Hillenbrand, L. A. 1997, AJ, 113, 1733

Hillenbrand, L. A., \& Hartmann, L. W. 1998, ApJ, 492, 540 
Jones, B. F., \& Walker, M. F. 1988, AJ, 95, 1755

Kounkel, M., Hartmann, L., Loinard, L., et al. 2014, ApJ, 790, 49

Kounkel, M., Hartmann, L., Loinard, L., et al. 2016a, ApJ, in press, arXiv: 1609.04041

Kounkel, M., Hartmann, L., Tobin, J. J., et al. 2016b, ApJ, 821, 8

Kukarkin, B. V., Kholopov, P. N., Pskovsky, Y. P., et al. 1971, General Catalogue of Variable Stars (3rd ed.; Moscow: Moscow State University)

Loinard, L. 2002, RMxAA, 38, 61

Loinard, L., Torres, R. M., Mioduszewski, A. J., et al. 2007, ApJ, 671, 546

McMullin, J. P., Waters, B., Schiebel, D., Young, W., \& Golap, K. 2007, adass XVI, 376, 127

Menten, K. M., \& Reid, M. J. 1995, ApJL, 445, L157

Menten, K. M., Reid, M. J., Forbrich, J., \& Brunthaler, A. 2007, A\&A, 474,515

Muench, A., Getman, K., Hillenbrand, L., \& Preibisch, T. 2008, in Handbook of Star Forming Regions, Vol. I, 4 (San Francisco, CA: ASP), 483

Muench, A. A., Lada, E. A., Lada, C. J., \& Alves, J. 2002, ApJ, 573, 366

Ortiz-León, G. N., Loinard, L., Dzib, S. A., et al. 2016, ApJ, in press, arXiv: 1611.06466

Parenago, P. P. 1954, TrSht, 25, 393
Petr, M. G., Coudé du Foresto, V., Beckwith, S. V. W., Richichi, A., \& McCaughrean, M. J. 1998, ApJ, 500, 825

Poveda, A., Allen, C., \& Hernández-Alcántara, A. 2005, ApJL, 627, L61

Poveda, A., Ruiz, J., \& Allen, C. 1967, BOTT, 4, 86

Pradel, N., Charlot, P., \& Lestrade, J.-F. 2006, A\&A, 452, 1099

Press, W. H., Teukolsky, S. A., Vetterling, W. T., \& Flannery, B. P. 1992, Numerical Recipes in FORTRAN. The Art of Scientific Computing (2nd ed.; Cambridge: Cambridge Univ. Press)

Reid, M. J., Menten, K. M., Zheng, X. W., et al. 2009, ApJ, 700, 137

Rieke, G. H., Low, F. J., \& Kleinmann, D. E. 1973, ApJL, 186, L7

Rivera, J. L., Loinard, L., Dzib, S. A., et al. 2015, ApJ, 807, 119

Rodríguez, L. F., Curiel, S., Cantó, J., et al. 2003, ApJ, 583, 330

Rodríguez, L. F., Dzib, S. A., Loinard, L., et al. 2017, ApJ, 834, 140

Rodríguez, L. F., Poveda, A., Lizano, S., \& Allen, C. 2005, ApJL, 627, L65

Schönrich, R., Binney, J., \& Dehnen, W. 2010, MNRAS, 403, 1829

Strand, K. A. 1958, ApJ, 128, 14

Vasilevskis, S. 1962, AJ, 67, 699

Vasilevskis, S. 1971, ApJ, 167, 537

Zapata, L. A., Rodríguez, L. F., Kurtz, S. E., \& O’Dell, C. R. 2004, AJ, 127,2252 